\title{
WEIGHTED MULTIPLE BIT-PLANE MATCHING, A SIMPLE AND EFFICIENT MATCHING CRITERION FOR ELECTRONIC DIGITAL IMAGE STABILIZER APPLICATION
}

\author{
H.R. Pourreza ${ }^{\dagger}$, M. Rahmati and F. Behazin \\ ${ }^{\dagger}$ Amirkabir University of Technology, Tehran, Iran \\ *YMA College, Tehran, Iran \\ pourreza,rahmati@ce.aku.ac.ir, behazin@ieee.org
}

\begin{abstract}
ABSTARCT
In this paper we propose two new matching criteria for template matching. The performance evaluation of these two criteria is applied to electronic digital image stabilizer (EDIS) application. These two criteria are based on bit-plane matching (BPM) criterion, where four decimated bit-planes are used in our criteria. These criteria can be realized using only Boolean functions; hence they can be realized very simple in any digital systems. We compared our criteria with other known criteria by employing twenty real video sequences.
\end{abstract}

Key words: Block matching, matching criterion, electronic digital image stabilization.

\section{INTRODUCTION}

The electronic digital image stabilization is the process of generating of compensated digital image sequence in which any unwanted camera motion is removed from the original sequence [pourr00a]. Using mechanical stabilizers based on accelerometers, gyros, or mechanical dampers is a traditional solution to the image stabilization problem, but these techniques are typically not precise and even after mechanical stabilization there may be significant residual image motion [Hanse94]. This shortcoming has led to the use of electronic digital image stabilizers. These image stabilizers use digital image processing techniques [Hanse94, Uomor90, Morim96, Ko98].

Digital image stabilization can generally be obtained in two basic stages I) motion estimation and II) motion correction (warping), as shown in Fig. 1. In feature-based stabilizers, several local motion vectors in different positions of an image, i.e. feature point (FP), are computed, and then global motion is estimated from these vectors [Ko98]. One method for local motion vector estimation is the use of block matching (BM) techniques [Morim96, Ko98]. In this technique, a block of $\mathrm{N} \times \mathrm{N}$ points is used for any feature point of current frame. By assuming that all pixels within the block have a uniform motion, for corresponding motion vector of the block, we search for a block on the previous frame that has the best match to it (according to a given criterion). The search performs within a larger block; i.e. $(N+2 w) \times(N+2 w)$; (search area) centered at the same location on the current frame, where $w$ denotes the maximum predicted displacement of any objects.

The block matching consists of two parts: choosing a BM criterion and selecting a search strategy. The accuracy of the estimation depends on both of these parts. The sum of squared difference (SSD) is the optimum criterion when additive noise distribution is Gaussian [Sebe00]. For cost effective realization of motion estimation, different algorithms have been proposed by researcher in order to reduce complexity of motion estimation [Pourr00b, Bhask97]. In this paper we present two simple and efficient matching criteria and then we compare them with some of the existing matching criteria. This paper is organized as follows. Some of existing matching criteria are introduced in section 2. In section 3 we explain our criteria that are Multiple Bit-Plane Matching (MBPM) and Weighted MBPM (WMBPM).

Some of the experimental results and conclusions are presented in section 4 and 5, respectively.

\section{THE MATCHING CRITERIA}

In this section we explain some of the important matching criteria in order that, we use to achieve the new criteria.

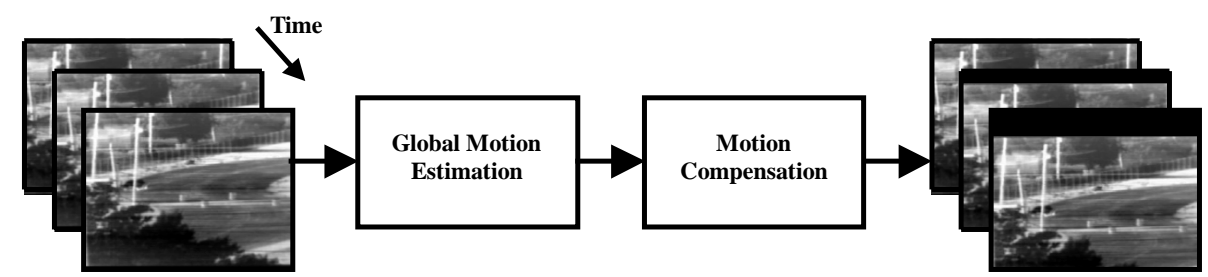

Fig. 1- Basic Structure of an EDIS 
We assume the additive noise has Gaussian distribution. The SSD is the optimum criterion when additive noise distribution was Gaussian. For two blocks, the SSD cost function for evaluation of matching at $(i, j)$ location of search area is as follows [Bhask97]:

$\operatorname{SSD}(i, j)$

$$
=\sum_{k} \sum_{l}(C(x+k, y+l)-P(x+i+k, y+j+l))^{2}
$$

where $C$ and $P$ are the current and previous frames pixels values respectively, $i$ and $j$ are defined in $-w \leq i \leq w$ and $-w \leq j \leq w,(x, y)$ denotes location of test block in image, and $(l, k) \in$ block area. We define as the best matching block, the block $P(x+i, y+l)$ for which $\operatorname{SSD}(i, j)$ is minimized. Thus $(u, v)(u=i$ and $v=j)$ represent the block motion vector.

In order to avoid square computation, sum of absolute difference (SAD) can be used as matching criterion (as SAD criterion is optimum for additive noise with exponential distribution [Sebe00], we use it as approximation of SSD). The SAD defined by [Bhask97]:

$$
\begin{aligned}
& S A D(i, j) \\
& \quad=\sum_{k} \sum_{l}|C(x+k, y+l)-P(x+i+k, y+j+l)|
\end{aligned}
$$

As in case of SSD, the motion vector $(u, v)$ corresponds with smallest $S A D$ within the search area.

The reduction of bit resolution of pixel values is one method for reducing the complexity of BM criterion [Bhask97]. The SAD and bit truncation method, as explain in [Chan95], can be used to improved hardware efficiency. Foe example we can use four most significant bits of 8-bit pixels for bit truncation.
As it called [Chan95], name of MPDC used for this criterion.

$$
\begin{aligned}
& \operatorname{MPDC}(i, j) \\
& \quad=\sum_{k} \sum_{l}\left|C_{B T}(x+k, y+l)-P_{B T}(x+i+k, y+j+l)\right|
\end{aligned}
$$

where $C_{B T}$ and $P_{B T}$ denote bit truncated of current and previous frames pixels value respectively.

For more simplicity, BPROP criterion used the XOR function instead of absolute difference in Eq. 3 [Bhask97]. The BPROP criterion defined by:

$$
\operatorname{BPROP}(i, j)
$$

$$
=\sum_{k} \sum_{l} X O R\left(C_{B T}(x+k, y+l), P_{B T}(x+i+k, y+j+l)\right)
$$

The simplest form of the bit resolution reduction is one bit per any pixel value, and this matching criterion is named bit-plane matching (BPM). In this method, both the current and the previous frames are transformed first into frames of binary-valued pixels. For two binary blocks, the $B P M$ cost function is defined by:

$\operatorname{BPM}(i, j)$

$$
=\sum_{k} \sum_{l} \operatorname{XOR}(\tilde{C}(x+k, y+l), \tilde{P}(x+i+k, y+j+l))
$$

where $\tilde{C}$ and $\tilde{P}$ denote current frame $(C)$ and previous frame $(P)$ pixels values respectively after they are transformed to one-bit frames. This means:

$$
\tilde{C}(i, j)=T[C(i, j)] \quad, \quad \tilde{P}(i, j)=T[P(i, j)]
$$

where $T[$.$] denotes a transformation.$

Ko et al [Ko98] proposed a simple transformation to compute the one-bit data. For the 8-bit gray-level of the pixel at location $(i, j)$ that can be represented as:

$$
f(i, j)=b_{7} 2^{7}+b_{6} 2^{6}+\ldots+b_{0} 2^{0}
$$

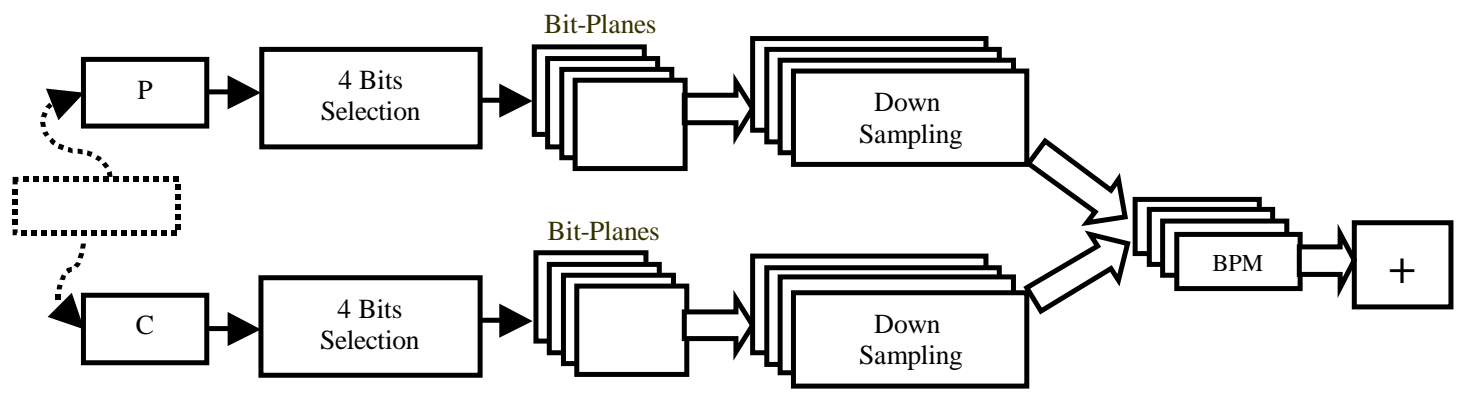

(a)

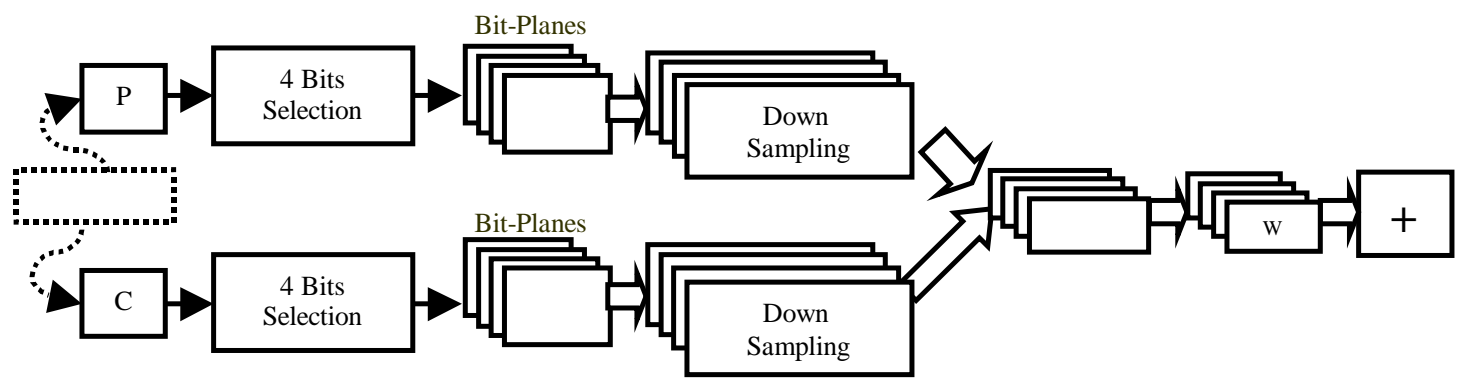

(b)

Fig. 2: (a) MBPM and (b) WMBPM criteria 
( $b_{k}$ is either 0 or 1 and $0 \leq k \leq 7$ ), Ko et al. proposed following transformation:

$T_{K o}[f(i, j)]=b_{4}$

Precision evaluation of motion estimation in our experiments shows that, the Ko's method for BPM (using $b_{4}$ ) does not yield proper results in different scenes and SNRs. We implemented Ko's method using several bits. In our experiments, the performance was achieved using $b_{4}$ or $b_{5}$ or $b_{6}$ for some cases of sequences. Therefore for some of the test sequences the $b_{4}$, other sequences $b_{5}$ and for remaining sequences $b_{6}$ present better performance. Therefore we present two new algorithms, as nonadaptive approaches, for binary transformation that have better performance than Ko method while they attain same computational complexity of it [Pourr00b].

\section{NEW CRITERIA}

We evaluated previously our proposed two new criteria for video compression application [Pourr00b]. These criteria are defined as following:

\subsection{MBPM $^{1}$ METHOD}

We compute the binary bit-plane by the transformation as following:

$T_{M B P M}[f(i, j)]=\left\{\begin{array}{l}b_{7}, \text { if }[\bmod (i, 2)=1 \text { and } \bmod (j, 2)=1] \\ b_{6}, \text { if }[\bmod (i, 2)=0 \text { and } \bmod (j, 2)=1] \\ b_{5}, \text { if }[\bmod (i, 2)=1 \text { and } \bmod (j, 2)=0] \\ b_{4}, \text { if }[\bmod (i, 2)=0 \text { and } \bmod (j, 2)=0]\end{array}\right.$

where $(i, j), 1 \leq i \leq N$ and $1 \leq j \leq N$, denotes coordination of $f$ on the block (Fig. 2.a). We refer to this BPM by Multiple Bit-Planes Matching $(M B P M)$.

\subsection{WEIGHTED MBPM (WMBPM)}

We define four transformations as following: $T_{W B B P M 1}[f(m, n)]$

$$
=\left\{\begin{array}{l}
b_{4}, \text { if }[\bmod (m, 2)=0 \text { and } \bmod (n, 2)=0] \\
0, \text { otherwise }
\end{array}\right.
$$

$T_{\text {WMBPM } 2}[f(m, n)]$

$$
=\left\{\begin{array}{l}
b_{5}, \text { if }[\bmod (m, 2)=1 \text { and } \bmod (n, 2)=0] \\
0, \text { otherwise }
\end{array}\right.
$$

$T_{W M B P M} 3[f(m, n)]$

$$
=\left\{\begin{array}{l}
b_{6}, \text { if }[\bmod (m, 2)=0 \text { and } \bmod (n, 2)=1] \\
0, \text { otherwise }
\end{array}\right.
$$

\footnotetext{
${ }^{1}$ Multiple Bit-Plane Matching
}

$$
\begin{aligned}
& T_{\text {WMBPM }}[f(m, n)] \\
& \quad=\left\{\begin{array}{l}
b_{7}, \text { if }[\bmod (m, 2)=1 \text { and } \bmod (n, 2)=1] \\
0, \text { otherwise }
\end{array}\right.
\end{aligned}
$$

Then, WMBPM defined as following:

$$
\begin{aligned}
\operatorname{WMBPM}(i, j)= & B P M_{\tilde{C}_{4}, \tilde{P}_{4}}(i, j)+2 B P M_{\tilde{C}_{5}, \tilde{P}_{5}}(i, j) \\
& +4 B P M_{\tilde{C}_{6}, \tilde{P}_{6}}(i, j)+8 B P M_{\tilde{C}_{7}, \tilde{P}_{7}}(i, j) \\
= & B P M_{\tilde{C}_{4}, \tilde{P}_{4}}(i, j)+2\left(B P M_{\tilde{C}_{5}, \tilde{P}_{5}}(i, j)\right. \\
& \left.+2\left(B P M_{\tilde{C}_{6}, \tilde{P}_{6}}(i, j)+2\left(B P M_{\tilde{C}_{7}, \tilde{P}_{7}}(i, j)\right)\right)\right)
\end{aligned}
$$

where $\tilde{C}_{k}$ and $\tilde{P}_{k}$ denote $C$ and $P$ under transformation of $T_{k}$, respectively, and $B P M_{\tilde{C}_{k}, \tilde{P}_{k}}$ denotes $B P M$ of $\tilde{C}_{k}$ and $\tilde{P}_{k}$ (Fig. 2.b). Motion vector $(u, v)$ correspond with smallest WMBPM within the search area.

\subsection{MBPM AND WMBPM FOR IMAGE STABILIZATION APPLICATION}

We evaluated our criteria for image stabilizing application. In this application, the accuracy of estimated motion vectors is very important. Thus, one of the stabilizer evaluation methods can be the evaluation of its motion estimator [Ko98]. We used the motion vectors obtained from the SSD criterion as reference and then calculated the root mean square error (RMSE) as following, for goodness evaluation of a criterion. The RMSE is given by:

$$
R M S E=\frac{1}{M} \sqrt{\sum_{m=1}^{M}\left(u_{m}-\hat{u}_{m}\right)^{2}+\left(v_{m}-\hat{v}_{m}\right)^{2}}
$$

where $\left(u_{m}, v_{m}\right)$ is the motion vector from the SSD, and $\left(\hat{u}_{m}, \hat{v}_{m}\right)$ is that from another criterion, and $M$ denotes total number of motion vectors.

Although the $b_{4}, b_{5}, b_{6}$ and $b_{7}$ bits for MBPM and WMBPM present the best results for video compression [Pourr00b] (Fig. 3), for image stabilization, we noticed that the $b_{3}, b_{4}, b_{5}$ and $b_{6}$ have the best performance. The results of our experiments presented in the next section.

\section{EXPERIMENTAL RESULTS}

20 popular sequences ${ }^{2}$ with a resolution of $176 \times 144$ pixels are used for our experiments. We used fixed 396 non-overlapped blocks as feature points in any frames and calculate motion vectors for these feature points by using $8 \times 8$ block size, $15 \times 15 \quad(w=7)$ search area and full search method respectively [Bhask97].

\footnotetext{
${ }^{2}$ We used these sequences: Akiyo, Bus, Carphone, Clair, Coastguard, Container, Flower, Football, Foreman, Grandma, Hallmonitor, Missamerica, Mobilecalender, News, Salesman, Silent, Stefan, Suzie, Tennis, and Trevor
} 
To evaluate precision of calculated motion vectors, we used RMSE as explained earlier (Sec. 3.3).

Our experiment accomplished on SSD, SAD, MPDC, BPROP, sub-sampled BPROP or BPROPS (4 to1 sub-sampling), Ko method (by using $b_{4}, b_{5}$, $b_{6}$ and $b_{7}$ ), MBPM and WMBPM (by using $b_{1} b_{2} b_{3} b_{4}, \quad b_{2} b_{3} b_{4} b_{5}, \quad b_{3} b_{4} b_{5} b_{6}$ and $b_{4} b_{5} b_{6} b_{7}$ bits $)$ matching criteria. The averages of RMSE for evaluated criteria on various sequences are shown in Fig. 4.

The SAD is uses 8 bit-per-sample (bps), MPDC and BPROP use 4bps, BPROPS uses $1 \mathrm{bps}$ in average and other criterion use 1 bps. The Fig. 4 shows that $\mathrm{WMBPM}_{3456}$ has the best performance among the $1 \mathrm{bps}$ criteria. While the result of $\mathrm{WMBPM}_{3456}$ is taken to be $100 \%$, the relative of RMSE for other criteria are calculated (in percent) and shown in the Table 1. The Ko $b 7$ method has maximum relative RMSE (168\%), and the WMBPM b2345 has the nearest RMSE. The computation burden of Ko and MBPM methods are same, while computation for WMBPM is further slightly [Pourr00b].

\section{CONCLUSIONS}

The MBPM and WMBPM presented as two simple and efficient matching criteria. These two criteria are based on bit-plane matching (BPM) criterion. Four decimated bit-planes are used in our criteria. Advantage of these two new criteria verified by conducting tests on 20 image sequences. The experimental results indicate that MBPM and WMBPM present better performance than other 1 bit-per-pixel algorithms.

\section{REFERENCES}

[pourr00a] Pourreza, H.R, Behazin, F. and Rahmati, M: A Wavelet-Based Template locating Criterion for Electronic Digital Image Stabilizer Application, Proc. Of IAPR Workshop on Machine Vision Applications, pp. 513-516, 2000.

[Hanse94] Hansen, M, Anandan, P, Dana, K, van der Wal, G, Burt, P: Real-time Scene Stabilization and Mosaic Construction, Proc. of the Second IEEE Workshop on Application of Computer Vision, pp. 54-62, 1994.

[Uomor90] Uomori, K, Morimora, A, Ishii, H, Sakaguchi, T. and Kitamura, Y: Automatic Image Stabilizing System by Full-Digital Signal

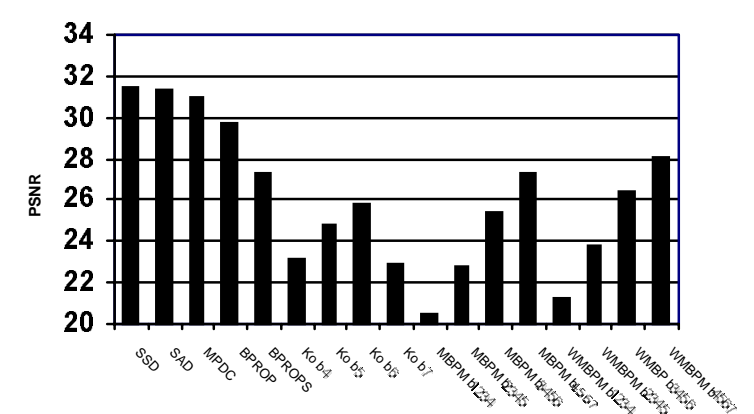

Fig. 3- PSNR(dB) versus matching criterion for twenty test sequences in video compression application

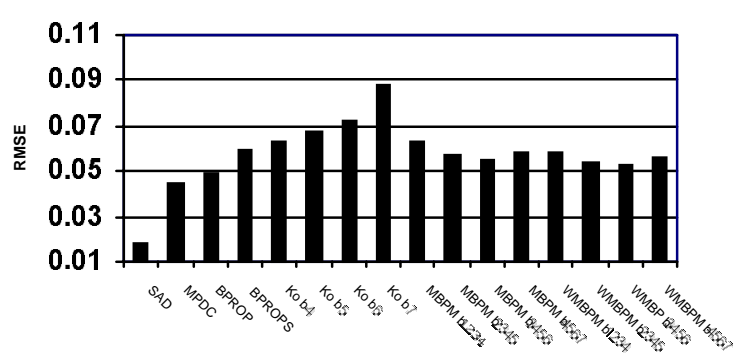

Fig. 4- RMSE versus matching criterion for twenty test sequences

Processing, IEEE Trans. On Consumer Electronics, Vol. 36, No. 3, pp. 510-519, 1990.

[Morim96] Morimoto, C, Chellappa, R: Fast Electronic Digital Image Stabilization, Proc. of IEEE ICPR 96, pp. 284-288, 1996.

[Ko98] Ko, S.J, Lee, S.H. and Lee, K.H: Digital Image Stabilizing Algorithms Based on BitPlane Matching, IEEE Trans. on Consumer Electronics, Vol. 44, No. 3, pp. 617-622, 1998.

[Sebe00] Sebe, N, Lew, M.S. and Huijsmans, D.P: Toward Improved Ranking Metrics, IEEE Trans. On Pattern Analysis and Machine Intelligence, Vol. 22, No. 10, pp. 1132-1142, 2000.

[Pourr00b] Pourreza, H.R, Rahmati, M. and Behazin, F: Simple and Efficient Bit-Plane Matching Algorithms for Video Compression, Proc. Of Workshop on Real-Time Image Sequence Analysis, pp. 33-42, 2000.

[Bhask97] Bhaskaran, V. and Konstantinides, K: Image and Video Compression Standards, Kluwer Academic Publishers, 1997.

[Chan95] Chan, Y, Kung, S.Y: Multi-Level Pixel Difference Classification Methods, Proc. Of IEEE Int. Conf. On Image Processing, pp. 252255, 1995.

Table 1- RMSE of 1bps Criteria versus $\mathrm{WMBPM}_{3456}$

\begin{tabular}{|l|l|l|l|l|l|l|l|l|l|l|l|l|}
\hline Criterion & $\begin{array}{l}\text { Ko } \\
\text { b4 }\end{array}$ & $\begin{array}{l}\text { Ko } \\
\text { b5 }\end{array}$ & $\begin{array}{l}\text { Ko } \\
\text { b6 }\end{array}$ & $\begin{array}{l}\text { Ko } \\
\text { b7 }\end{array}$ & $\begin{array}{l}\text { MBPM } \\
\text { b1234 }\end{array}$ & $\begin{array}{l}\text { MBPM } \\
\text { b2345 }\end{array}$ & $\begin{array}{l}\text { MBPM } \\
\text { b3456 }\end{array}$ & $\begin{array}{l}\text { MBPM } \\
\text { b4567 }\end{array}$ & $\begin{array}{l}\text { WBBPM } \\
\text { b1234 }\end{array}$ & $\begin{array}{l}\text { WBBPM } \\
\text { b2345 }\end{array}$ & $\begin{array}{l}\text { WBBPM } \\
\text { b4567 }\end{array}$ & $\begin{array}{l}\text { BPROPS } \\
112 \%\end{array}$ \\
\hline & $121 \%$ & $128 \%$ & $137 \%$ & $168 \%$ & $120 \%$ & $109 \%$ & $105 \%$ & $111 \%$ & $112 \%$ & $102 \%$ & $107 \%$ & $114 \%$ \\
\hline
\end{tabular}

